\title{
Erratum: Distinguishing black holes from horizonless objects through the excitation of resonances during inspiral [Phys. Rev. D 100, 084046 (2019)]
}

Vitor Cardoso®, Adrián del Río, and Masashi Kimura

(Received 8 March 2020; accepted 10 March 2020; published 20 March 2020)

DOI: $10.1103 /$ PhysRevD.101.069902

Equation (A39) in our paper has a typo; it should read

$$
\dot{E}_{s=0}^{l=m=1} \approx \frac{\gamma^{2} m_{0}^{2} M^{2}}{24 \pi r_{p}^{4}}
$$

This typo propagated to Eq. (3). Taking into account also the $l=-m=1$ mode, the correct Eq. (3) should read

$$
\dot{E}_{s=0}=\frac{\gamma^{2} m_{0}^{2} M^{2}}{12 \pi r_{p}^{4}}
$$

This is the leading order term in a post-Newtonian expansion. Such expression is in perfect agreement with previous results in the literature [1-3].

\section{ACKNOWLEDGMENTS}

We are indebted to Francisco Duque for a careful reading of the original manuscript, and for bringing the typo to our attention. V. C. would like to thank Waseda University for warm hospitality and support while this work was finalized. V. C. acknowledges financial support provided under the European Union's H2020 ERC Consolidator Grant "Matter and strongfield gravity: New frontiers in Einstein's theory" grant Agreement No. MaGRaTh-646597. This project has received funding from the European Union's Horizon 2020 research and innovation programme under the Marie Sklodowska-Curie grant Agreement No. 690904. We thank FCT for financial support through Project No. UIDB/00099/2020. We acknowledge financial support provided by FCT/Portugal through grant PTDC/MAT-APL/30043/2017. The authors would like to acknowledge networking support by the GWverse COST Action CA16104, "Black holes, gravitational waves and fundamental physics."

[1] V. Cardoso, M. Cavaglia, and J. Q. Guo, Phys. Rev. D 75, 084020 (2007).

[2] V. Cardoso, S. Chakrabarti, P. Pani, E. Berti, and L. Gualtieri, Phys. Rev. Lett. 107, 241101 (2011).

[3] N. Yunes, P. Pani, and V. Cardoso, Phys. Rev. D 85, 102003 (2012).

Published by the American Physical Society under the terms of the Creative Commons Attribution 4.0 International license. Further distribution of this work must maintain attribution to the author(s) and the published articles title, journal citation, and DOI. 